\title{
Segmentación y valoración del mercado turístico: Estudio del destino Patrimonio de la Humanidad Trinidad de Cuba
}

\author{
García Reinoso, Nelson ${ }^{1}$
}

\begin{abstract}
Resumen
El presente artículo tiene como objetivo identificar los perfiles de los diferentes segmentos de turistas que visitan el destino turístico Trinidad de Cuba, declarada Patrimonio de la Humanidad por la UNESCO en 1998. La metodología parte de un análisis factorial exploratorio y luego una clasificación basada en un algoritmo mixto: clasificación jerárquica con el método de Ward y agregación alrededor de centros móviles (K-medias), se realizó una encuesta aplicada por cuestionario a 484 turistas y excursionistas que visitan el destino por motivaciones turísticas. Los resultados obtenidos permitieron la identificación de dos segmentos de turistas: Turismo cultural patrimonial y Turismo de Naturaleza, que según los resultados de los test estadísticos, ambos grupos difieren significativamente en cuanto a sus rasgos demográficos; características del viaje; relación con el producto o servicio (motivaciones) y evaluación y fidelidad del destino. Este artículo brinda información relevante a actores territoriales involucrados en el turismo, para que puedan mejorar la calidad de sus productos y servicios. Permite renovar productos segmentados para alcanzar un mejor posicionamiento turístico en correspondencia con los perfiles de turistas y el mercado objetivo.
\end{abstract}

Palabras clave: segmentación de mercado; turismo cultural; motivaciones turísticas; trinidad de Cuba.

Recibido: 28.11.18 Aceptado: 20.06.19

1 Máster en Gestión Turística (UCLV-Cuba). Doctorando en Turismo EDUA-Universidad de Alicante (España). Docente investigador, Carrera de Turismo de la Escuela Superior Politécnica Agropecuaria de Manabí "Manuel Félix López" (ESPAM MFL). Coordinador Maestría en Turismo ESPAM, Email: nelsongreinoso@gmail.com 


\title{
Segmentation and valuation of the tourist market: Study of the destination World Heritage Trinidad de Cuba
}

\begin{abstract}
:
The objective of this article is to identify the profiles of the different segments of tourists that visit the tourist destination Trinidad de Cuba, declared a World Heritage Site by UNESCO in 1998. The methodology is based on an exploratory factor analysis and then a classification based on a mixed algorithm: hierarchical classification with the Ward method and aggregation around mobile centers (K-means), a questionnaire survey was applied to 484 tourists and hikers visit the destination for tourist reasons. The results obtained allowed the identification of two segments of tourists: Heritage Cultural Tourism and Nature Tourism, which according to the results of the statistical tests, both groups differ significantly in terms of their demographic characteristics; travel characteristics; relationship with the product or service (motivations) and evaluation and fidelity of the destination. This article provides relevant information to territorial actors involved in tourism, so that they can improve the quality of their products and services and; it allows to renew segmented products to reach a better tourist positioning in correspondence with the profiles of tourists and the target market.
\end{abstract}

Keywords: Market segmentation; Cultural tourism; Tourist motivations; Trinidad of Cuba.

\section{Introducción}

El creciente desarrollo del turismo cultural en los últimos años se debe fundamentalmente segúnJiménez et al, (2009: 400-401)a "la valoración creciente de la cultura y el patrimonio histórico por parte de la sociedad, (...) este crecimiento ha sido más notable en las ciudades o conjuntos históricos declarados Patrimonio de la Humanidad”."En (...) Cuba, la cultura ha sido el eje articulador del desarrollo social, económico y ambiental de las áreas históricas"(Leal Spengler, 2006:13). Estas distinciones convierten a Cuba en el tercer destino de Latinoamérica en cuanto a ciudades patrimoniales, solo después de México y Brasil(Guilarte, 2015)."Desde hace décadas es creciente la incorporación de las ciudades patrimoniales a los itinerarios turísticos"(Rodríguez \& AbdulJalbar, 2012:42). Cuba cuenta con un destacado patrimonio cultural que se evidencia en las cuatro ciudades históricas que han sido declaradas patrimonio cultural de la humanidad, ellas son La Habana (1982), Trinidad (1988), Cienfuegos (2005) y Camagüey (2008).

Los destinos patrimoniales (...), tienen que afrontar de forma rigurosa y sistemática aquellos aspectos vinculados con la inserción equilibrada de la actividad 
turística(Brito, 2007). Consideración que conduce a la necesidad de interpretar vías de solución más adecuada(Reinoso, 2017)a problemas o necesidades que interfieren en el desarrollo local. Los actuales escenarios(Narváez \& Fernández, 2013) originan la necesidad de que se adopten estrategias que permitan dar respuestas a los desafíos y a las demandas de entonos dinámicos, cambiantes y complejos;y orientar su acción hacia los objetivos previstos en los planes de desarrollo local(Narváez et al, 2018); donde la puesta en valor del patrimonio natural, cultural, social, arquitectónico y gastronómico de la localidad es preponderante(Reinoso \& Doumet, 2017).

El desarrollo turístico en Cuba se ha basado históricamente en el modelo de sol y playa, sin embargo, la fuerte competencia en el Caribe y las exigencias actuales de la demanda reclaman la búsqueda de otras alternativas turísticas(Guilarte, 2015). Coincidiendo con Mustelier \& García (2015:7-8)"el desarrollo del turismo de Cuba (...)se sustenta en la modalidad de sol y playa".El turismo cultural(Guilarte, 2015), podría constituir una importante alternativa para Cuba, que diversifica su producto tradicional de sol y playa(García \& Garcés, 2008), para abarcar diversas formas de turismo de ciudad e histórico cultural.

La inclusión del centro histórico de Trinidad y el Valle de los Ingenios en el listado de bienes materiales protegidos por la UNESCO considerados Patrimonio Cultural de la Humanidad (Reinoso \& Quintero, 2018) constituye una fortaleza del municipio para el desarrollo de la actividad turística. Trinidad pose el centro histórico colonial mejor conservado de Cuba y del Caribe, y vincula su atractivo colonial de su arquitectura y urbanismo en un entorno de potencialidades; en su enclave geográfico entre la montaña y el litoral (Martínez \& Díaz, 2011).

La importancia de conocer el perfil de los usuarios de un destino(García et al, 2011)radica en la posibilidad de comprender mejor las razones de visita y el uso que hacen del lugar los visitantes(Meiriño, Brea, Vila, \& López, 2016). Y según Mendoza et al, (2017) permite direccionar las gestiones estratégicas de marketing y promoción turística.Los factores determinantes o que condicionan la decisión de viajar están agrupados según su naturaleza en: Económicos, relativos a las unidades demandantes, sistema de comercialización y satisfacción del cliente (Narváez \& Fernández, 2010; Alatorre \& Pérez, 2011).

La segmentación del mercado tiene como finalidad(Gouvêa \& Niño, 2004), encontrar similitudes entre grupos acerca del producto que se les está ofreciendo y encontrar rasgos básicos del consumidor de manera que responderán de manera similar a las estrategias de comercialización implantadas.De esta manera(LópezGuzmánet al, 2016), la oferta puede responder mejor a sus necesidades y expectativas con la finalidad de conseguir una mayor satisfacción por parte de los diferente grupos.

El objetivo planteado en esta investigación consistió en identificar los perfiles de los diferentessegmentos de turistas que visitan el destino turístico Trinidad de Cuba, a través de una segmentación del mercado. En este sentido, se coinciden con los criterios metodológicos deCollado et al, (2007) al establecer dos objetivos específicos: 1) estudiar la situación del turismo en Trinidad desde la óptica de la demanda turística; y 2) identificar segmentos de 
turistas mediante sus motivaciones; el análisis de las diferencias en sus características contribuirán a mejorar las estrategias de comercialización y promoción del destino para cada uno de los segmentos.

En este contexto el artículo aborda el estudio de las motivaciones que estimulan al turista a elegir un determinado destino. La metodología cuantitativa aplicada a través de una encuesta realizada en el destino, partió del estudio de cuatro aspectos fundamentales: rasgos demográficos; características del viaje; relación con el producto o servicio (motivaciones) y evaluación y fidelidaddel destino. Mediante un crece de variables (análisis por conglomerados) y considerando las motivaciones como eje de análisis, fue posible definir dos segmentos de turistas y establecer el perfil del visitante para el destino Trinidad de Cuba.

\section{Segmentación del mercado a partir de las motivaciones turísticas.}

Los estudios de turismo sobre segmentación psicográfica(Stylidis et al, 2018) se han centrado con frecuencia en los turistas como unidad de análisis y dos explicaciones sostenibles incluyen que: i) desempeñan el papel de "contribuyente de ingresos" que influye en la salud económica de un lugar de destino; y ii) los antecedentes de los turistas que visitan un lugar de destino suelen ser diversos. Investigaciones previas sobre motivaciones turísticas(Andreu et al, 2005:2) evidencian que la evaluación de los atributos del destino puede diferir de un destino a otro dependiendo del tipo de destinos y motivaciones turísticas. La investigación de razones o motivaciones para viajar(Park \& Yoon, 2009:100) contribuye a comprender el turismo como un fenómeno social y psicológico y ofrece perspectivas de gestión prácticas.

La literatura sobre turismo enfatiza la importancia de los factores de atracción y empuje en la configuración de la motivación turística y la elección de los destinos de vacaciones. Crompton, (1979)explora los factores motivadores de empujar para describir las elecciones y el comportamiento de los turistas: la búsqueda de conocimiento, la relajación y la unión familiar se conocen como factores de empuje, mientras que las preferencias por entornos naturales e históricos, costos, instalaciones, seguridad, y la accesibilidad se informa como factores de atracción(Jang \& Wu, 2006 y Rid et al, 2014)

Estas motivaciones se han clasificado de acuerdo con la siguiente tipología por Goeldner y Ritchie (2003): (i) físico, como la relajación; (ii) culturales, como el descubrimiento de nuevas áreas geográficas; (iii) interpersonales, como socializar y conocer gente nueva; y (iv) el prestigio, la autoestima (Park \& Yoon, 2009:100)

El conocimiento sobre las motivaciones turísticas (Prebensen et al, 2010:859)juega un papel importante en el desarrollo de planes de mercadeo efectivos.Se consideran determinantes de las actividades turísticas (Albayrak \& Caber, 2018: 202) con respecto a (i) las razones para viajar, (ii) por qué se selecciona un destino específico, (iii) y la satisfacción general con el viaje. De acuerdo con (Hsu et al, 2010) "la motivación de las personas para realizar una actividad es una función de la expectativa de que serán capaces de realizar la actividad y obtener los resultados deseados, y el valor personal 
de todos los resultados asociados con esa actividad".

Esta perspectiva(Albayrak \& Caber, 2018: 202) permite a los investigadores aclarar qué motivaciones relacionadas con los valores y expectativas afectan las decisiones de viaje y la satisfacción de los turistas. La evaluación de las motivaciones turísticas se utiliza (Andreu et al, 2005) para comprender mejor las opciones, preferencias y necesidades de los visitantes.

En el campo de la investigación sobre el comportamiento y el consumo turístico, se detecta una tendencia a incorporar la segmentación (González \& Molina, 2007)como una herramienta complementaria a otras técnicas de análisis en el estudio de conexiones causales entre dimensiones teóricas, adoptando la siguiente secuencia: reducción de datos (técnicas factoriales); la clasificación (conglomerados) y explicación (técnicas de dependencia).

La segmentación de los consumidores parte del supuesto de que los mercados son intrínsecamente heterogéneos, y que la preferencia de los consumidores varía conforme sus valores, necesidades, deseos, restricciones, creencias e incentivos(Añaña et al, 2017). Precisamente para la obtención de estos perfiles o segmentos, los expertos en marketing utilizan los llamados criterios de segmentación(BeltránBueno \& Parra-Meroño, 2017) que pueden ser muy diversos (demográficos, socioeconómicos, motivacionales, psicográficos, entre otros). Al mismo tiempo, los productos (incluidos los destinos turísticos) compiten entre sí para satisfacer las necesidades y deseos de los consumidores.

Es posible agrupar los métodos de segmentación en dos grupos: la segmentación a priori o predeterminada, el criterio o criterios de selección de los segmentos se establece previamente a la recogida de los datos(Prado \& Mallou, 2000; González \& Molina, 2007), es establecida por el investigador anticipadamente en base a las características demográficas y psicográficas(Beltrán-Bueno, 2014) y la segmentación post hoc o a posteriori, se utiliza cuando se desconocen las características del mercado y, para ello, se realiza, en primer lugar, un análisis cualitativo que permita conocer el merado y, posteriormente, se aplica un análisis de conglomerados que permite determinar los segmentos de mercado más relevantes y diferentes (BeltránBueno, 2014); emplea un conjunto amplio de variables para definir los segmentos en función de su homogeneidad respecto a dichas variables (González \& Molina, 2007), la cual es ejecutada a partir del análisis de clúster basado en la similitud de algunos atributos de interés(Añaña et al, 2017).

El análisis factorial exploratorio (AFE) es uno de los métodos estadísticos que se utiliza de manera frecuente en la investigación en ciencias sociales y seguramente es una de las técnicas que más decisiones requiere por parte del investigador a la hora de ejecutar dicho análisis (Frías- Navarro \& Soler, 2012). De hecho, el análisis factorial se ha utilizado ampliamente en la investigación de segmentación de visitantes (Park \& Yoon, 2009:102). El objetivo del Análisis Factorial exploratorio (AFE) es eliminar la información redundante, obteniendo como resultado los factores, que reflejan la asociación de las variables originales (Pérez \& Medrano 2010; Brida et al, 2012; Lloret-Segura et al, 2014; Martínez-González, 2014).Es un método multivariante que pretende 
expresar $p$ variables observables como una combinación lineal de $m$ variables hipotéticas o latentes, denominadas factores(Cuadras, 2019).

Tiene una formulación parecida al Análisis de Componentes Principales, pero el modelo que relaciona variables y factores es diferente en AFE. Si la matriz de correlaciones existe, las componentes principales también existen, mientras que el modelo factorial podría ser aceptado o no mediante un test estadístico.También el investigador dispone de algunas pruebas que le permiten evaluar el grado de adecuación de sus datos(Frías- Navarro \& Soler, 2012), como por ejemplo el test de esfericidad de Bartlett o el índice de medida de adecuación de la muestra KMO(Frías- Navarro \& Soler, 2012; Hair et al, 2014; Arcentales et al, 2016; Ramirez-Anormaliza et al, 2017).

El análisis de conglomerados - clúster permite conseguir una clasificación o agrupamiento de los individuos en "n" grupos según el comportamiento de éstos en una serie de segmentos se ha utilizado el análisis de conglomerados (clúster). Es una técnica multivariante que busca agrupar elementos o variables tratando de lograr la máxima homogeneidad en cada grupo y la mayor diferencia entre ellos, mediante una estructura jerarquizada para poder decidir qué nivel jerárquico es el más apropiado para establecer la clasificación. Permite clasificar una población en un número pequeño de grupos mutuamente exclusivos y exhaustivos, sin tener en cuenta las posibles dependencias entre las variables, es decir, no pretende explicar estadísticamente ningún hecho concreto(Vilà-Baños et al, 2014; BeltránBueno \& Parra-Meroño, 2017). El objetivo será formar el mínimo número de grupos posible, lo más homogéneos dentro de cada grupo, y lo más heterogéneos entre grupos(Ricolfe et al, 2008; Lopes et al, 2010).

\section{Algunas precisiones metodológicas}

La presente investigación tiene como objetivo identificar los perfiles de los diferentes segmentos de turistas que visitan el destino turístico Trinidad de Cuba, a través de una segmentación del mercado. La investigación es cuantitativa, con un enfoque descriptivocorrelacional. El análisis de los antecedentes metodológicos que se relacionan con el tema parte de la selección de diferentes metodologías para el estudio de mercado turístico, que buscan la identificación de los perfiles de los visitantes en el turismo, dentro de los que destacan los siguientes autores: García et al, (2011); Lopes, (2011); Bonilla et al, (2014); García et al, (2017) y Calvo, (2017). Se estableció un procedimiento metodológico (ver cuadro1) que se relaciona y tributa al cumplimiento del objetivo planteado en la presente investigación.

Las Técnicas de análisis utilizadas han sido básicas (medias y frecuencias), tabulación cruzada, análisis de la varianza, análisis factorial, segmentación (análisis clúster) y análisis discriminante (Ricolfe et al, 2008). El procedimiento permite realizar primero un análisis factorial exploratorio según la naturaleza que presentan los datos (Pardo \& Del Campo, 2007) y luego una clasificación basada en un algoritmo mixto: clasificación jerárquica con el método de Ward y agregación alrededor de centros móviles (K-medias), considerado, generalmente, como el 


\section{Cuadro 1. \\ Procedimiento metodológico para el estudio del mercado turístico.}

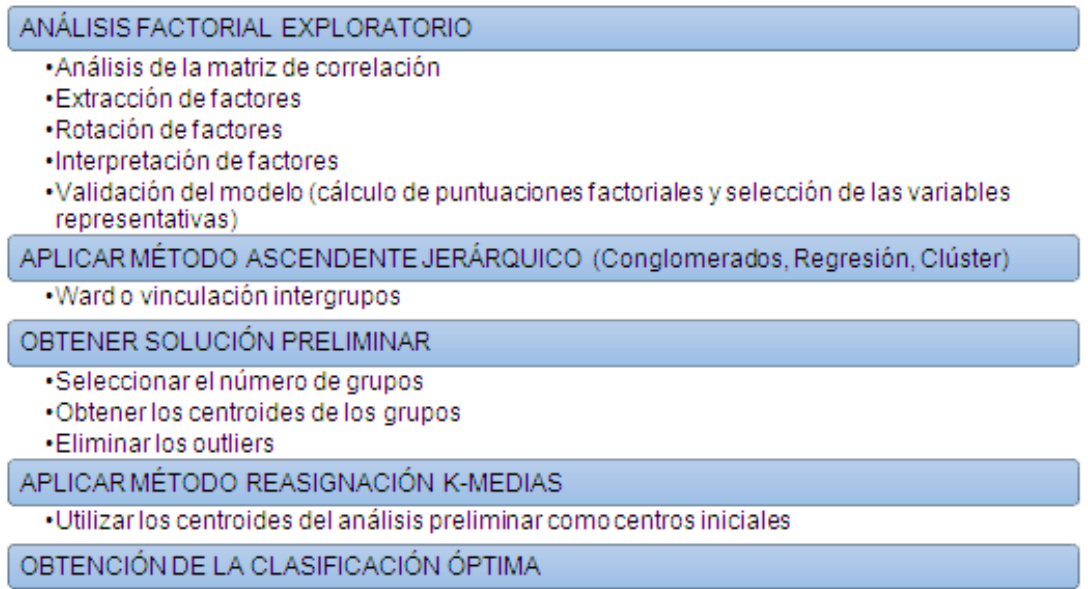

Fuente: elaboración propia a partir de Aranda et al, (2002); Pardo \& Del Campo, (2007); García et al, (2011); Lopes, (2011); Bonilla et al, (2014); García et al, (2017) y

(Ramirez-Anormaliza et al, 2017).

método más robusto disponible en el paquete estadístico SPSS (Lopes et al, 2010).

El método de recogida de datos es el muestreo aleatorio sistemático (se escoge un primer individuo al azar y el resto vienen condicionado por una cifra fija, en este caso, cada 2 individuos). Para determinar el tamaño de la muestra se utiliza la metodología propuesta porAlatorre \& Pérez, (2011), asumiendo una población infinita. La variabilidad de la población se estima en un $50 \%(p=q=$ $0,5)$ (Meiriño et al, 2016). Se recopilaron 484 encuestas válidas, representando un error muestral del $4,56 \%$ para un nivel de confianza del 95,5\% (Tabla 1).

\section{Tabla 1}

Ficha técnica de la investigación

\begin{aligned} & \hline \multicolumn{1}{c}{ Características } \multicolumn{1}{c}{ Encuestas } \\ & \hline Universo $\begin{array}{l}\text { Turistas y excursionistas mayores de } 18 \text { años que visitan Trinidad de } \\ \text { Cuba, por motivaciones turísticas. }\end{array} \\ &$ Ámbito Geográfico Trinidad de Cuba. \\ & Metodología Encuesta personal mediante cuestionario estructurado \\ & Diseño muestral Muestreo aleatorio sistemático \\ & \hline\end{aligned}


Segmentación y valoración del mercado turístico: Estudio del destino Patrimonio de la Humanidad Trinidad de Cuba

García Reinoso, Nelson

\section{Cont... Tabla 1}

\begin{tabular}{|c|c|c|}
\hline Error de muestreo & \multicolumn{2}{|l|}{$4,56 \%$} \\
\hline Nivel de confianza & \multicolumn{2}{|c|}{$95,5 \% \quad(z=2,0047) \quad(p=q=0,5)$} \\
\hline $\begin{array}{r}\text { Tamaño de la mues- } \\
\text { tra }\end{array}$ & \multicolumn{2}{|l|}{484 encuestas } \\
\hline $\begin{array}{r}\text { Fecha del trabajo de } \\
\text { campo }\end{array}$ & \multicolumn{2}{|c|}{15 de diciembre de 2017 al 7 de enero del 2018} \\
\hline \multicolumn{3}{|c|}{$\begin{array}{l}\text { Fuente: Elaboración propia a partir deCristóbal-Fransi et al,(2014) } \\
\qquad \text { Agüera et al, (2017) y Franco et al, (2017) }\end{array}$} \\
\hline $\begin{array}{l}\text { El trabajo de cam } \\
\text { la encuesta estructura } \\
\text { de } 19 \text { ítems basado } \\
\text { (Collado et al, 2007;N } \\
\text { 2007; Ricolfe et al, 2008 } \\
\text { 2009;García et al, } 2 \text { } \\
\text { Vázquez de la Torre et } \\
\text { al, 2017; Beltrán-Buer } \\
\text { 2017; Franco et al, } \\
\text { 2017;Carvache-Francc } \\
\text { Trigo et al, 2018)donde } \\
\text { de factores que deter } \\
\text { el perfil del cliente, }\end{array}$ & $\begin{array}{l}\text { poy de aplicación de } \\
\text { por un cuestionario } \\
\text { en estudios previos } \\
\text { lolera \& Albaladejo, } \\
\text {; Jiménez \& Vargas, } \\
11 \text {;Pesonen, 2014; } \\
\text { al, 2016; Agüera et } \\
\text { o \& Parra-Meroño, } \\
\text { 2017; García et al, } \\
\text { et al, 2018;Prada- } \\
\text { se pregunta acerca } \\
\text { minan la demanda, } \\
\text { de satisfacción y }\end{array}$ & $\begin{array}{l}\text { turísticos del territorio. } \\
\text { La estructura seguida en el } \\
\text { cuestionario (ver diagrama 1) está dividido } \\
\text { en cuatro bloques: (1) direccionado a } \\
\text { las características demográficas; (2) las } \\
\text { características del viaje, donde destacan } \\
\text { los factores que motivaron su visita, (3) } \\
\text { relacionado con el servicio o producto; } \\
\text { y el (4) y último bloque a las evaluación } \\
\text { y satisfacción, con el objetivo obtener } \\
\text { información concerniente a la valoración de } \\
\text { la oferta turística así como la satisfacción y } \\
\text { fidelización de los turistas. }\end{array}$ \\
\hline
\end{tabular}
valoración de los productos y servicios

\section{Diagrama 1 \\ Listado de variables de segmentación}

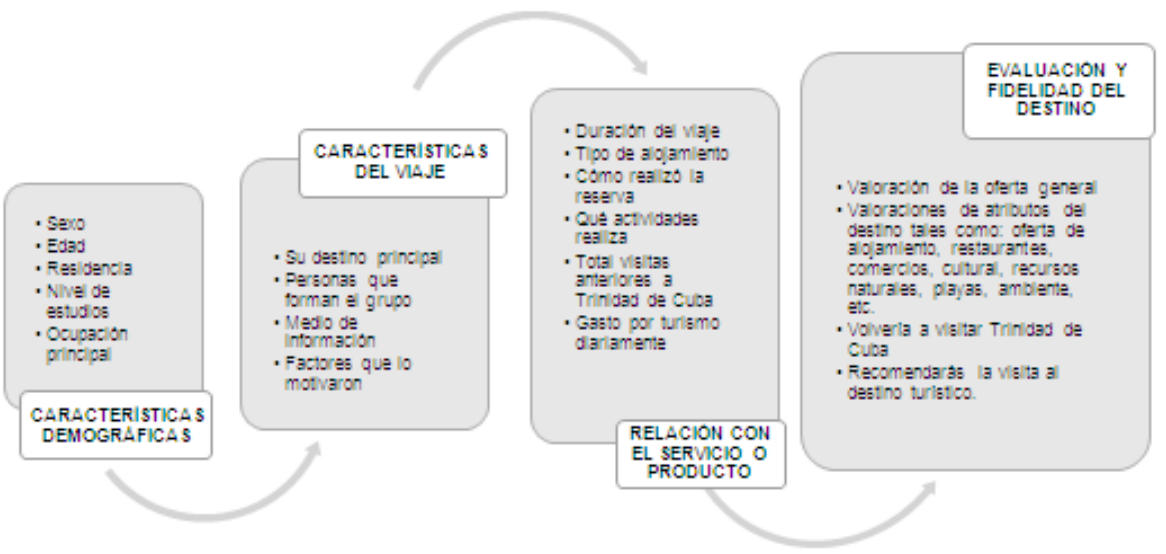

Fuente: Elaboración propia. 


\section{Caracterización del turismo cultural en Trinidad de Cuba}

Trinidad, fundada oficialmente como Villa de la Santísima Trinidad y llamada por algunos historiadores y habitantes como La Trinidad o Trinidad de Cuba. Es una ciudad ubicada en la región central de Cuba, específicamente en el sur de la provincia Sancti Spíritus, y es la capital del municipio del mismo nombre. La Villa de la Santísima Trinidad fue la tercera villa fundada por la corona española en Cuba, el 10 de enero de 1514, se fundó con la presencia de Diego Velázquez de Cuéllar, y fue evolucionando con rapidez, lo cual le posibilitó ser una de las más prósperas de Cuba.

Trinidad y el Valle de los Ingenios quedaron inscritos en 1988 como patrimonio cultural, la ciudad debe su nacimiento a la industria azucarera y constituye un ejemplo representativo del urbanismo de los primeros asentamientos fundados en América en el siglo XVI, y de su armónico crecimiento hasta inicios del siglo XIX. Entre los atractivos de la denominada Ciudad Museo de Cuba sobresalen el recorrido por sus empedradas calles, la Plaza Mayor y la Iglesia Mayor de la Santísima Trinidad.

El Valle de los Ingenios es otro paisaje que muestra el auge de la actividad azucarera dentro del sistema colonial español en América. Trinidad se distingue por ser el principal destino turístico del centro sur de Cuba, con sus inigualables valores arquitectónicos, que clasifican como uno de los conjuntos mejor conservados de la etapa colonial en América. Junto a las empedradas calles y plazuelas, amplias casonas, palacios y palacetes, museos, casasmuseo y otras instituciones culturales.
Trinidad se presenta como un modelo turístico de carácter integral (Canales \& Sabariegos, 2011) al conjugar tres ámbitos geográficos diferentes: el urbano, el costero y el de monte; todo bajo un criterio de sostenibilidad que es el que se viene desarrollando en el país desde que éste se abre al turismo internacional, concepto convertido en objetivo que proyectan a todos los sectores donde se desarrolla el turismo en la isla.

El polo turístico de Trinidad, uno de los más completos de Cuba, se caracteriza por una excelente combinación de turismo de sol y playa, con las playas Ancón, María Aguilar y La Boca, y sus hoteles Ancón, Costasur y Brisas Trinidad del Mar. Aguas adentro se encuentra Cayo Blanco, en cuya costa sur existe una pequeña playa de gran belleza, de arenas blancas y aguas cristalinas. Hacia el oeste de Cayo también existen grandes colonias de coral a profundidades entre 18 y 40 metros.

"En Trinidad se conjugan como en pocos lugares de Cuba elementos físicos, naturales, históricos y culturales que son ideales para el desarrollo de las múltiples modalidades del turismo, (...) que confieren a Trinidad ser un destino turístico integral" (Rodríguez \& López, 2011:51). Trinidad es conocida como la "ciudad de los museos» (...) esta oferta museística refuerza el carácter cultural de la población (Canales \& Sabariegos, 2011: 104).

Trinidad conserva una valiosa parte de su patrimonio histórico en cada museo. Cada uno de ellos constituye una reliquia arquitectónica y cultural de la villa más colonial de Cuba. Los museos de Trinidad son únicos en el país, el Museo de Arquitectura Colonial de Trinidad ilustra el desarrollo que alcanzó 
la villa en la etapa colonial, el visitante se encuentra directamente con los estilos de vida, el mobiliario y la decoración propios de las construcciones coloniales cubanas y latinoamericanas. Destacan otros museos como el Nacional de Lucha contra Bandidos, el museo Municipal de Trinidad, el Romántico de Trinidad uno de los más visitados en Cuba.

Trinidad declarada el 30 de julio de 2018 con la condición de Ciudad Artesanal del Mundo, refleja un ejemplo del rescate y florecimiento de las tradiciones artesanales, donde más de mil artesanos trabajan la cestería y otras artes manuales heredadas de sus antepasados, expresiones que constituyen un modo de subsistencia de varias generaciones de trinitarios. El municipio en la actualidad muestra un acelerado crecimiento del sector turístico acompañado de las nuevas necesidades y motivaciones por parte de la demanda, lo que provoca una transformación del desarrollo de la actividad en diferentes ámbitos, en especial en lo referente a la comercialización turística, la cual debe enfocarse principalmente en las necesidades de cada nicho de mercado para su posterior satisfacción, así como de igual manera atraer un mayor número de demanda.

\section{Segmentación y valoración del mercado turístico: Resultados de la descripción de la muestra}

Las estadísticas de arribo de turismo internacional al destino Trinidad proyectan un crecimiento positivo, lo que expone un destino en desarrollo, en el año 2010 arribaron 311116 clientes y en el 2015 se alcanza la cifra de 383092 turistas (Oficina Nacional de Estadística Cuba, 2016). Destacan como principales mercado emisores en el año 2015 los siguientes países: Alemania (76649); Francia (53620); Canadá (47604); Italia (16576); Inglaterra (15890) y Estados Unidos (11882); entre otros. Cabe destacar que en el año anterior (2017) el mercado estadounidense tuvo un importante crecimiento, significando casi el $45 \%$ de los clientes del último trimestre del año (2016).

Se realizó un trabajo de campo que consistió en la aplicación de 484 encuestas las mismas que contenían variables destacadas. Los datos fueron analizados en tres etapas. Primero, se aplicó un análisis estadístico descriptivo a los datos recopilados para explorar el perfil general de la muestra (Tabla 2).

Tabla 2

Principales características demográfica y del viaje de la muestra (porcentaje de la muestra)

\begin{tabular}{lrrrr}
\hline \multicolumn{2}{c}{ Características demográfica } & $\%$ & Características del viaje & $\%$ \\
& & & & \\
\hline Residencia $(\mathrm{N}=478)$ & Francia & $19 \%$ & Su destino principal $(\mathrm{N}=478)$ \\
& Holanda & $17 \%$ & Trinidad & $53,5 \%$ \\
& Alemania & $16 \%$ & La Habana & $14 \%$ \\
& EEUU & $14 \%$ & Varadero & $8,8 \%$ \\
\hline
\end{tabular}




\title{
Cont... Tabla 3
}

\begin{abstract}
Actividades realizadas $(\mathrm{N}=484)$ (Puntuación media en una escala ascendente de 10 puntos, desde muy insatisfactorio a muy satisfactorio): Ir a la playa (7.8), Realizar excursiones senderismo (7.6), Tours por la cuidad (7.2), Visitar museos (7), Visitar casco urbano (6.4), Visitar sitios turísticos próximos (5.8).

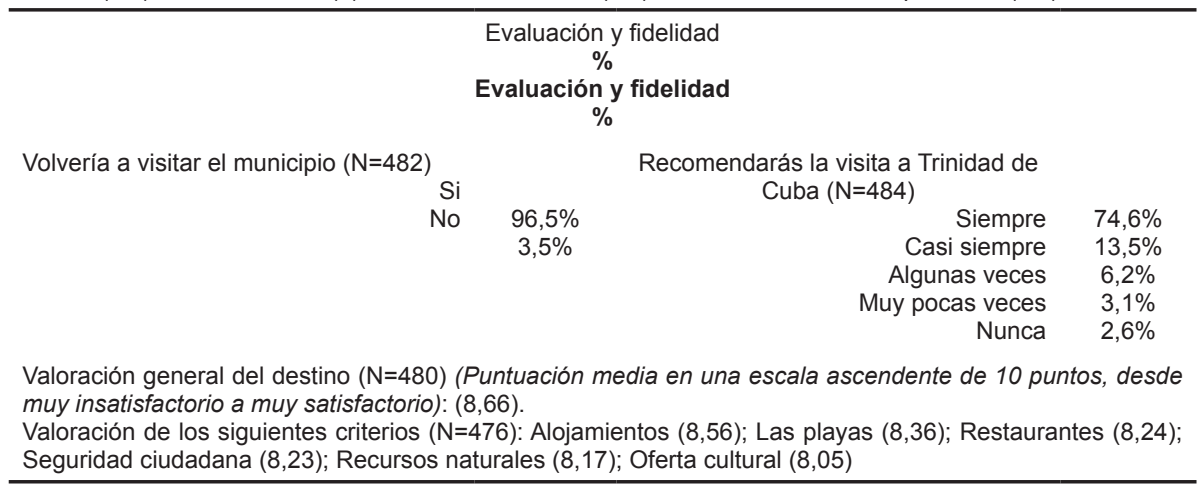

Fuente: Elaboración propia.

Es importante destacar que dentro del tipo de alojamiento destaca el Hotel (54\%) seguido de la Hostal (38\%); que la duración del viaje en un mayor porciento es de 3 a 6 noches (44,5\%), y se reserva por medio de una agencia de viajes (44\%). La muestra evidencia una media de 8.66 puntos (satisfactoria) en la evaluación general y fidelidad con el destino turístico de Trinidad de Cuba

\section{Segmentación del mercado turístico en el destino Trinidad de Cuba}

Esta investigación en segundo lugar aplica un análisis de componentes principales (ACP) con rotación varimax, como la elección adecuada entre los métodos de estimación del análisis factorial exploratorio (AFE). Este análisis se aplicó a trece ítems para medir las dimensiones de motivación que subyacen en la elección de los turistas de las actividades turísticas. Este estudio utilizó los criterios sugeridos por (Park \& Yoon, 2009:102): (1) carga del factor igual o superior a 0.50 , (2) valores propios iguales o superiores a 1.0, y (3) los resultados del análisis factorial explican al menos el $72,46 \%$ de la varianza total (tabla 4). Se descartaron todos aquellos ítems que mostraban correlaciones muy débiles, inferiores a 0,3 (Cea, 2002; De Vaus, 2002 y Brida et al, 2012). 


\begin{tabular}{|c|c|c|}
\hline \multicolumn{3}{|c|}{$\begin{array}{c}\text { Tabla } 4 \\
\text { Matriz de componentes rotados }{ }^{a}\end{array}$} \\
\hline \multirow[b]{2}{*}{ Motivaciones turísticas } & \multicolumn{2}{|c|}{ Componente } \\
\hline & 1 & 2 \\
\hline La naturaleza, paisaje & ,862 & \\
\hline El clima &, 728 & \\
\hline El poder ir a la playa &, 617 & \\
\hline El precio &, 523 & \\
\hline El patrimonio cultural e histórico & & ,833 \\
\hline Me gusta el Caribe & &, 701 \\
\hline$\%$ de la varianza explicada & 39,896 & 32,570 \\
\hline$\%$ acumulado de la varianza & 39,896 & 72,467 \\
\hline
\end{tabular}

Fuente: elaboración propia basada en el método de extracción: Análisis de componentes principales. Método de rotación: Normalización Varimax con Kaiser

Se calculó una medida de KaiserMayer-Olkin a 0.874 , y la prueba de esfericidad de Bartlett mostró una gran significación ( $p<0.000$ ), lo que demuestra una alta adecuación para realizar un análisis factorial con los elementos elegidos (Rid et al, 2014:106) as in many other African countries, rural areas rarely profit from the turnover earned in the country's tourism sector. In academic and political literature, however, rural tourism is frequently identified as a diversification strategy that may trigger local economic development in remote communities. To promote rural tourism development, further knowledge is required to understand why tourists are motivated to engage in distinct tourism market segments. In this study, survey data was collected from 450 tourists in The Gambia using a self-administered questionnaire. The questionnaire was constructed to identify the key characteristics and motivations of tourists so that the significant market segments could be categorized and the (latent.

En la investigación en tercer lugar se realiza un análisis de conglomerados, se pasan a identificar los segmentos empleando las variables anteriormente seleccionadas. Primero, los grupos de turistas se formaron sobre la base de las puntuaciones de los dos factores, utilizando el método de Ward. Se obtuvo una solución de dos grupos basada en un examen del coeficiente de aglomeración para el análisis de grupos jerárquicos (Andreu et al, 2005). El segundo paso utiliza técnicas no jerárquicas (es decir, algoritmo K-medias) para ajustar los resultados de los procedimientos jerárquicos (Park \& Yoon, 2009:102). El resultado final del análisis de conglomerados ha conducido a la identificación de dos grupos (ver tabla 5), solución que se consideró apropiada porque proporcionaba los segmentos más interpretables. 


\begin{tabular}{|c|c|c|c|c|c|}
\hline \multirow[b]{3}{*}{ Motivaciones Turísticas } & \multirow{2}{*}{\multicolumn{2}{|c|}{$\begin{array}{l}\text { Rango promedio (Prueba U de } \\
\text { Mann-Whitney) }\end{array}$}} & \multirow[b]{3}{*}{ Media Muestra } & \multirow{2}{*}{\multicolumn{2}{|c|}{ U de Mann-Whitney }} \\
\hline & & & & & \\
\hline & $\begin{array}{l}\text { Turismo cultural } \\
\text { patrimonial }\end{array}$ & $\begin{array}{l}\text { Turismo de } \\
\text { Naturaleza }\end{array}$ & & Estadístico & $\begin{array}{l}\text { Sig. Asintótica } \\
\text { (bilateral) }\end{array}$ \\
\hline El Clima & 67,56 & 136,19 & 7,2000 & 1566,000 & 0,000 \\
\hline El poder ir a la playa & 117,87 & 81,69 & 8,3700 & 3186,000 & 0,000 \\
\hline La naturaleza, paisaje & 67,45 & 136,31 & 7,7000 & 1554,000 & 0,000 \\
\hline $\begin{array}{r}\text { El patrimonio cultural e } \\
\text { histórico }\end{array}$ & 116,95 & 82,68 & 7,8700 & 3281,500 & 0,000 \\
\hline Me gusta el Caribe & 104,11 & 96,59 & 5,3500 & 4617,000 & 0,323 \\
\hline El precio & 116,85 & 82,79 & 5,5000 & 3292,000 & 0,000 \\
\hline
\end{tabular}

Nota: 1 nada importante, 10 muy importante. Kaiser - Meyer - Olkin medida de la adecuación del muestreo 0.874. Chi-cuadrado 193,178 - Prueba de esfericidad de Bartlett,

$$
p<0,000 \text {. }
$$

Fuente: elaboración propia

Se identificaron los perfiles de clientes para cada uno de los dos segmentos identificados; se utilizan las tablas de contingencias entre variables de interés (Garcia \& Gallard, 2002) que permite relacionarlas pues conocer las características de cada uno es sumamente importante para poder identificar las mejores estrategias. La relación con el servicio o producto de los conglomerados obtenidos, sobre la base de la muestra total, (ver tablas 6), se aplicaron una serie de pruebas de $U$ de Mann-Whitney para investigar si hubo diferencias estadísticas entre las variables de comportamiento turístico de cada grupo que visitó Trinidad de Cuba.

\section{Tabla 6}

\section{Relación con el servicio o producto por cada segmento}

\begin{tabular}{rccccc}
\hline $\begin{array}{c}\text { Relación con el servicio o producto } \\
(\%)\end{array}$ & $\begin{array}{c}\text { Clúster 1 } \\
\text { Turismo cultural } \\
\text { patrimonial }\end{array}$ & $\begin{array}{c}\text { Clúster 2 } \\
\text { Turismo de } \\
\text { Naturaleza }\end{array}$ & $\begin{array}{c}\text { Muestra } \\
(\%)\end{array}$ & $\begin{array}{c}\text { U de Mann-Whitney } \\
\text { Estadístico }\end{array}$ & $\begin{array}{c}\text { Sig. asin- } \\
\text { tótica }\end{array}$ \\
\hline Duración del viaje & & & & 2644,000 & 0,000 \\
Excursión 1 día & 2,9 & 10,4 & 6,5 & & \\
1 a 2 noches & 6,7 & $\mathbf{5 7 , 3}$ & 31,0 & & \\
3 a 6 noches & $\mathbf{7 2 , 1}$ & 14,6 & 44,5 & & \\
7 o más noches & 18,3 & 17,7 & 18,0 & & \\
¿Qué tipo de alojamiento haces? & & & & 1878.000 & \\
Vivienda amigo / familiar & 4,8 & 1,0 & 3,0 & & \\
Hotel & $\mathbf{8 1 , 7}$ & 24,0 & 54,0 & & \\
\hline
\end{tabular}




\section{Cont... Tabla 6}

\begin{tabular}{|c|c|c|c|c|c|}
\hline Hostal & 12,5 & 65,6 & 38,0 & & \\
\hline Camping & 1,0 & 9,4 & 5,0 & & \\
\hline ¿Cómo reservó? & & & & 2482,000 & 0,000 \\
\hline Directamente & 51,0 & 12,5 & 32,5 & & \\
\hline Sin reserva & 26,0 & 16,7 & 21,5 & & \\
\hline Por agencia de viajes & 19,2 & 70,8 & 44,0 & & \\
\hline Otro & 3,8 & 0,0 & 2,0 & & \\
\hline $\begin{array}{l}\text { Total de visitas anteriores a Tri- } \\
\text { nidad }\end{array}$ & & & & 3096,000 & 0,000 \\
\hline Nunca & 26,0 & 69,8 & 47,0 & & \\
\hline 4 o más visitas & 28,8 & 11,5 & 20,5 & & \\
\hline 2 a 3 visitas & 37,5 & 3,1 & 21,0 & & \\
\hline $1 \mathrm{vez}$ & 7,7 & 15,6 & 11,5 & & \\
\hline $\begin{array}{l}\text { ¿Cuánto gastas por turismo } \\
\text { diariamente (se excluye aloja- } \\
\text { miento)? }\end{array}$ & & & & 2129,000 & 0,000 \\
\hline Hasta $\$ 40$ (dólares americanos) & 1,0 & 24,0 & 12,0 & & \\
\hline De $\$ 41$ a $\$ 70$ & 7,7 & 34,4 & 20,5 & & \\
\hline De $\$ 71$ a $\$ 100$ & 60,6 & 34,4 & 48,0 & & \\
\hline Más de $\$ 101$ & 30,8 & 7,3 & 19,5 & & \\
\hline
\end{tabular}

Fuente: elaboración propia

A partir de su análisis, se puede concluir que existen diferencias ( $p$ $<0.05$ ) en la composición de los grupos en relación con el Duración del viaje ( $p<0.000$ ), ¿Qué tipo de alojamiento haces? ( $p<0.000)$, ¿Cómo reservó? $(p<0.000)$, total de visitas anteriores a Trinidad $(p<0,000)$ y ¿Cuánto gastas por concepto de turismo diariamente (se excluye alojamiento)? ( $p<0,000)$.

Los hallazgos del estudio muestran dos segmentos de turistas: i) Turismo cultural patrimonial y ii) Turismo de Naturaleza. Estos segmentos se identifican en relación con las motivaciones y presentan diferentes perfiles de comportamiento de viaje. Los resultados también confirman que, como se ha sugerido en la literatura sobre turismo cultural, la mayoría de los visitantes que se identifican por el turismo cultural están caracterizado por clientes con elevado interés por conocer sitios Patrimonio de la Humanidad y destaca que un $75 \%$ prefieren a Trinidad como destino principal.

De igual manera se encuentran diferencias significativas en las motivaciones del viaje, el clúster Turismo cultural patrimonial prefiere la playa, el patrimonio cultural e histórico, el Caribe, el precio y la tranquilidad; mientras que para el Turismo de Naturaleza lo es el clima, la restauración, el ocio nocturno, la naturaleza y el paisaje. Enmarcadas son las diferencias en la relación con el 
producto o servicio, el clúster cultural identifica clientes que se alojan en hoteles, con estancias de 3 a 6 noches, que hacen la reserva directamente pues la mayoría han visitado el destino anteriormente; el clúster naturaleza se alojan mayoritariamente en Hostales, con una duración de 1 a 2 noches y la mayoría reserva a través de una agencia de viaje pues por lo general nunca han visitado Trinidad.

Estas diferencias persisten evaluación del destino y la fidelidad, en este aspecto en cuanto a la intención de regresar, resulto como aspecto negativo que un $14 \%$ de los visitantes segmentados en el grupo de turismo de naturaleza no regresaría a Trinidad. Por otra parte, conviene destacar que el primer segmento es el mayoritario, representando un $52 \%$ de los encuestados.

\subsection{Evaluación de atributos del destino}

Las valoraciones medias de cada grupo sobre los principales aspectos que constituyen el destino turístico se muestra en la Tabla 7. Los resultados del análisis de la varianza indican que los dos grupos difieren significativamente en las valoraciones de los atributos del destino.

Tabla 7

\section{Evaluación de atributos del destino}

\begin{tabular}{|c|c|c|c|c|c|}
\hline \multirow{2}{*}{$\begin{array}{l}\text { Evaluación de atributos del des- } \\
\text { tino }\end{array}$} & \multicolumn{2}{|c|}{ Rango promedio } & \multirow{2}{*}{$\begin{array}{l}\text { Media } \\
\text { Muestra }\end{array}$} & \multicolumn{2}{|c|}{ U de Mann-Whitney } \\
\hline & $\begin{array}{l}\text { Turismo cultural } \\
\text { patrimonial }\end{array}$ & $\begin{array}{l}\text { Turismo de } \\
\text { Naturaleza }\end{array}$ & & Estadístico & $\begin{array}{l}\text { Sig. asin- } \\
\text { tótica }\end{array}$ \\
\hline $\begin{array}{r}\text { ¿Cómo valoras la oferta turística } \\
\text { general de Trinidad? }\end{array}$ & 124,65 & 74,33 & 8,6650 & 2480,000 & 0,000 \\
\hline La oferta de alojamientos & 116,82 & 77,36 & 8,5692 & 2823,500 & 0,000 \\
\hline La oferta de restaurantes & 97,33 & 101,85 & 8,2475 & 4669,000 & 0,566 \\
\hline Oferta de ocio nocturno & 76,02 & 86,43 & 7,9441 & 2816,000 & 0,147 \\
\hline Oferta de comercios & 68,91 & 134,04 & 7,8342 & 1706,500 & 0,000 \\
\hline $\begin{array}{r}\text { Oferta cultural y monumental } \\
\text { (museos) }\end{array}$ & 121,14 & 69,94 & 8,0518 & 2178,500 & 0,000 \\
\hline Oferta náutica & 67,67 & 120,05 & 7,6284 & 1780,500 & 0,000 \\
\hline Oferta deportiva & 68,29 & 127,03 & 7,5368 & 1713,000 & 0,000 \\
\hline Seguridad ciudadana & 120,22 & 78,31 & 8,2362 & 2861,500 & 0,000 \\
\hline Servicios de información turística & 124,16 & 67,66 & 7,5885 & 1895,500 & 0,000 \\
\hline Recursos naturales & 71,11 & 130,28 & 8,1768 & 1968,500 & 0,000 \\
\hline La dotación de playas & 75,07 & 128,05 & 8,3650 & 2347,500 & 0,000 \\
\hline Limpieza del municipio & 124,08 & 74,96 & 7,4750 & 2540,000 & 0,000 \\
\hline Renta de autos y transporte & 128,88 & 69,76 & 5,7350 & 2041,000 & 0,000 \\
\hline El ambiente & 70,41 & 133,09 & 7,9900 & 1863,000 & 0,000 \\
\hline
\end{tabular}

Fuente: Elaboración propia. 


\section{Conclusiones}

La importancia de conocer el perfil de los usuarios de un destino radica en la posibilidad de comprender mejor las razones de visita y el uso que hacen del lugar los visitantes. Al identificar los perfiles de los diferentes segmentos de turistas que visitan el destino turístico Trinidad de Cuba, se utilizó como variable de segmentación las motivaciones turísticas, su medición es la clave para satisfacer a los visitantes actuales, atraer a más visitantes, predecir la demanda futura y desarrollar productos para satisfacer las necesidades de los turistas.

Las motivaciones de turismo cultural patrimonial y turismo de naturaleza tuvieron el puntaje más alto para aquellos que visitan Trinidad de Cuba. Este resultado también se corresponde con los hallazgos de una encuesta realizada por el Ministerio de Turismo de Cuba en el año 2016, referenciadas y analizadas en artículos publicado por los autores (Canales \& Sabariegos, 2011) y (Reinoso \& Quintero, 2018).

Identificar las motivaciones o las razones para elegir un destino en particular como criterios de medición del desempeño podría proporcionar valiosas implicaciones para la gestión de destinos a fin de identificar el perfil de sus clientes reales, por ejemplo, valores, preferencias y comportamiento. Esta es una razón potencial y práctica por la cual es esencial explorar los determinantes de las motivaciones y variaciones de los turistas entre los distintos segmentos. En un mercado en continuo crecimiento como se evidencia en el destino Trinidad de Cuba, las estrategias de comercialización nacen de las necesidades de la demanda las cuales cambian constantemente y la prioridad es conocerlas para cubrirlas y poder satisfacer a esas necesidades aspecto que se alcanza solo a través una adecuada segmentación del mercado turístico.

Los turistas no siempre asignan la misma importancia a los atributos del producto, por lo que es crucial entender los factores que influyen en el comportamiento del turista y qué elementos particulares consideran los turistas como importantes. De acuerdo a los perfiles de la demanda que visita el destino Trinidad de Cuba, se pudo corroborar que existen diferencias significativas en cuanto a la valoración y evaluación, que hacen del destino en su conjunto, como en sus atributos; los visitantes agrupados en el turismo cultural patrimonial hacen una mejor valoración general del destino en su conjunto (99\% regresaría y un $98 \%$ recomienda el destino), destacando el valor que le dan a los alojamientos, la oferta cultural y monumental, la seguridad ciudadana, la información turística, limpieza del municipio y renta de autos y transporte. Sin embargo los visitantes identificados en el grupo de turismo de naturaleza emiten una valoración menos satisfactoria del destino (un 14\% nunca regresaría y un $3.4 \%$ no lo recomienda), aunque valoran mejor su oferta de restaurantes, ocio nocturno, comercios, náutica, deportiva, recursos naturales, la dotación de playas, y el ambiente.

Además de las contribuciones teóricas, este estudio también proporciona una serie de implicaciones prácticas para las autoridades locales, los emprendedores y los planificadores de turismo; ofrece un enfoque perspicaz y válido para identificar la existencia de dos grupos de turistas distintos que muestran actitudes disímiles hacia el 
turismo La segmentación del mercado turístico pretende contribuir a mejorar las estrategias de comercialización y promoción del destino turístico Trinidad de Cuba, para cada uno de los segmentos detectados. La heterogeneidad detectada en las motivaciones del viaje, pone de relieve que la oferta turística y las estrategias de comercialización deben orientarse hacia aquellos segmentos de consumidores que más valoren los atractivos que constituyen ventajas diferenciales de la zona: fundamentalmente los aspectos culturales; patrimoniales y naturales del territorio.

Las limitaciones de esta investigación se centran en primer lugar, en que los hallazgos proporcionados por el presente estudio son perspicaces pero no exhaustivos. Dado que las teorías de la motivación turística deben ser dinámicas y flexibles en todas las situaciones y las circunstancias individuales de los turistas, siempre hay más oportunidades de investigación para este concepto complejo. $Y$ en segundo lugar, la encuesta se realizó durante una temporada alta de vacaciones (Semana Santa y actividades de fin de año) y, por lo tanto, el momento podría haber influido significativamente en las percepciones de los turistas sobre el destino Trinidad de Cuba.

Como futuras líneas de investigaciones se pueden explorar hasta qué punto la temporada alta o baja de turismo influye en la motivación de viajar y en la imagen positiva $y$ negativa que tienen los turistas sobre un destino turístico. Se podría utilizar una nueva encuesta para estudiar si existen diferencias significativas entre los turistas que visitan el destino Trinidad de Cuba en diferentes épocas del año.

\section{Referencias bibliográficas}

Agüera, Francisco, Salvador Cuadra, Tomás López-Guzmán, y Pablo Morales. (2017), Estudio de La Demanda Existente En Torno Al Oleoturismo. El Caso de Andalucía. Cuadernos de Turismo Pp. 39: 437-53.

Alatorre, Alejandra, y Manuel Pérez. (2011), Procedimiento Para Medir La Demanda Turística En Un Destino. Revista de Investigación En Turismo y Desarrollo Local TURyDES 4 (11): Pp. 1-25.

Albayrak, Tahir, \& Meltem Caber. (2018), Examining the Relationship between Tourist Motivation and Satisfaction by Two Competing Methods. Tourism Management 69 (June): Pp. 201-13.

Añaña, Edar, Francisco dos Anjos, y Melise Pereira. (2017), Segmentación Post-Hoc del mercado turístico en los destinos del litoral Brasileño. Análisis según los valores personales de los turistas. Estudios y Perspectivas En Turismo 26: Pp. 662-77.

Andreu, Luisa, Metin Kozak, Nilgun Avci, y Nurten Cifter. (2005), Market Segmentation by Motivations to Travel: British Tourists Visiting Turkey. Journal of Travel \& Tourism Marketing 19 (1): Pp. 1-14.

Aranda, Francisco, Pilar Delgado, y Pilar Urbistondo. (2002), Imagen Turística de Las Comunidades Autónomas Españolas Ante Una Demanda Segmentada. Estudios de Economía Aplicada 20 (3): Pp. 627-49.

Arcentales, Evelin, Faviola Carpio, Jesmy González, y Huber Vázquez. (2016), Desarrollo turístico: estudio empírico de la población económicamente activa del cantón Milagro. PODIUM 29: Pp. 109-20.

Beltrán-Bueno, Miguel Á. (2014), Las 
motivaciones como factor clave de la conducta turística: un estudio empírico en el sureste Español. Universidad Católica de Murcia.

Beltrán-Bueno, Miguel Á., y María C. Parra-Meroño. (2017), Perfiles turísticos en función de las motivaciones para viajar. Cuadernos de Turismo 39: Pp. 41-65.

Bonilla, Jesús M., Andreea Boerasu, y Luis M. Bonilla. (2014), Perfiles de los visitantes en el turismo de naturaleza: análisis exploratorio en el entorno de Doñana. International Journal of World of Tourism 1 (1): Pp. 17-25.

Brida, Juan G., Eugenia Riaño, y Sandra Aguirre. (2012), Percepciones de los residentes acerca de los impactos del turismo de cruceros en la comunidad: un análisis factorial y de clústeres. Cuadernos de Turismo 29: Pp 79-107.

Brito, Marcelo. (2007), Las ciudades históricas como destinos patrimoniales: potencialidades y requisitos. Ediciones de la Fundació Càtedra Iberoamericana. Palma de Mallorca.

Calvo, María I. (2017), Identificación de los perfiles dinámicos provinciales de la demanda turística en España. Un enfoque exploratorio espacio-temporal. Revista Investigaciones Turísticas 14: Pp. 63-86.

Canales, Gregorio, y José Sabariegos. (2011), Trinidad, producto turístico integral y sostenible en Cuba. Cuadernos de Turismo No. 27: Pp. 95-114.

Carvache-Franco, Mauricio, Wilmer Carvache-Franco, Cristina López, y Miguel Mejía. (2018), Motivaciones, valoración y satisfacción del turista en un destino de sol y playa de Ecuador. Revista Espacios 39 (13): Pp. 3-16.
Cea, M. A. (2002), Análisis multivariable. Teoría y práctica en la investigación social. Madrid: Síntesis.

Collado, Arturo, David Navarro, Águeda Talaya, y Estrella Sánchez. (2007), Segmentación de la demanda turística: un análisis aplicado a un destino turístico cultural. Revista de Análisis Turístico 4 (2): Pp. 36-48.

Cristóbal-Fransi, Eduard, Natalia Daries-Ramon, y Juan Pablo Baldomar. (2014), Segmentación de los e-consumidores: un estudio aplicado a partir de las perspectivas de uso de internet. Cuadernos de Gestión 14 (1): Pp. 35-55.

Crompton, John L. (1979), Motivation for pleasure vacation. Annals of Tourism Research 6: Pp. 408-24.

Cuadras, Carles M. (2019), Nuevos métodos de análisis multivariante. Barcelona, Spain: CMC Editions.

Franco, Wilmer, Mónica Torres-Naranjo, y Mauricio Franco. (2017), Análisis del perfil y satisfacción del turista que visita Montañita-Ecuador. Cuadernos de Turismo 39: Pp. 113-29.

Frías- Navarro, Dolores, y Marcos Soler. (2012), Prácticas del análisis factorial exploratorio (afe) en la investigación sobre conducta del consumidor y marketing. Suma Psicológica 19 (1): Pp. 47-58.

Garcia, Carme, y Germa Gallard. (2002), Segmentación del mercado turístico según las preferencias ambientales. Cuadernos de Turismo 9: Pp. 123-35.

García, Maribel, Lourdes E. Amores, Rocío del Carmen Barquín, y Irma Y. Soto. (2017), Perfil del visitante de naturaleza en Latinoamérica: prácticas, motivaciones e imaginarios. Estudio comparativo entre México y Ecuador. Pasos Revista de Tur- 
Segmentación y valoración del mercado turístico: Estudio del destino Patrimonio de la Humanidad Trinidad de Cuba

García Reinoso, Nelson

ismo y Patrimonio Cultural 15 (3): Pp. 713-29.

García, Maribel, Sergio Maass, Irma Ramírez de la O, Gabino Bernal, Gerardo N. Espinosa de los Monteros, y Héctor H. Regil. (2011), El visitante del Parque Nacional Nevado de Toluca, México. Análisis del comportamiento en un área natural protegida. Investigaciones Geográficas 76: Pp. 56-70.

García, Robiel, y Yunia Garcés. (2008), "Turismo y desarrollo local. Proyecto para convertir «Cocodrilo» en pueblo turístico sostenible en un área protegida de La Isla de La Juventud (Cuba). Cuadernos de Turismo 22: Pp. 9-33.

González, Pablo, y Oscar Molina. (2007), La segmentación de la demanda turística Española. Metodología de Encuestas 9: Pp. 57-92.

Gouvêa, María, y Fanny Niño. (2004). Marketing Estratégico: segmentación y posicionamiento en agencias de viaje y turismo de la ciudad de São Paulo - Brasil. Estudios y Perspectivas En Turismo 13: Pp. 240-57.

Guilarte, Yamilé. (2015), La imagen del turismo cultural en Cuba percibida por el mercado Español. Turismo y Sociedad XVI: Pp. 67-84.

Hair, Joseph, William Black, Barry Babin, \& Rolph Anderson. (2014), Multivariate data analysis. Seventh Ed. United States of America: Pearson Education Limited.

Hsu, Cathy H.C., Liping A. Cai, \& Mimi Li. (2010), "Expectation, motivation, and attitude: a tourist behavioral model. Journal of Travel Research 49 (3): Pp. 282-96.

Jang, Soo Cheong, \& Chi Mei Emily Wu. (2006), Seniors' Travel motivation and the influential factors: an examination of Taiwanese Seniors. Tourism Management 27 (2): Pp. 306-16.

Jiménez, José, y Manuel Vargas. (2009), Construcción de un modelo para el análisis de motivaciones sobre la elección de un destino turístico. Estudios y Perspectivas En Turismo 18: Pp. 400-413.

Jiménez, Juan A., José Jiménez, Claudia Sevilla, y María Rodríguez. (2009), La fundación turismo de Cuenca: un nuevo modelo de gestión pública y privada. Turismo y Patrimonio Cultural 7 (2): Pp. 281-96.

Leal Spengler, Eusebio. (2006), La Cultura, única certeza para un proyecto sostenible. In Manejo y Gestión de Centros Históricos. La Habana Vieja, Cuba: Ediciones Boloña.

Lloret-Segura, Susana, Adoración Ferreres-Traver, Ana Hernández-Baeza, y Inés Tomás-Marco. (2014), El análisis factorial exploratorio de los ítems: una guía práctica, revisada y actualizada. Anales de Psicología 30 (3): Pp. 1151-69.

Lopes, Sérgio D. (2011), Geo-Segmentación y Geo-Posicionamiento en el análisis de las preferencias de los turistas: la geometría al servicio del marketing. Estudios y Perspectivas En Turismo 20: Pp. 842-54.

Lopes, Sérgio D., Antonio Boubeta, y Jesús Mallou. (2010), Segmentación Post Hoc del mercado turístico Español. Aplicación de análisis clúster en dos etapas. Estudios y Perspectivas En Turismo 19: Pp. 592-606.

López-Guzmán, Tomás, Claudia P. Uribe, Guzmán Muñoz-Fernández, y Ingrid Ríos. (2016), Perfil sociodemográfico, valoración de atributos y nivel de satisfacción. Estudio de festivales gastronómicos, Guayaquil - Ecuador. Revista Venezolana de 
Gerencia 21 (76): Pp. 674-92.

Martínez-González, Miguel Á. (2014), Bioestadística amigable. Barcelona, España: GEA Consultoría Editorial, s.l.

Martínez, Gregorio, y José Díaz. (2011), "Trinidad, producto turístico integral y sostenible en Cuba." Cuadernos de Turismo 27: Pp. 95-114.

Meiriño, Roberto, José A. Brea, Noelia Vila, y Elena López. (2016), Segmentación del mercado de un destino turístico de interior. El caso de A Ribeira Sacra (Ourense). PASOS. Revista de Turismo y Patrimonio Cultural 14 (2): Pp. 369-83.

Mendoza, Ángel G., Sandra E. Jiménez, Juan J. Noguera, y Maribel Ramos. (2017), Recuperación de destinos turísticos posterremoto: revisión del caso Ecuador. Revista Venezolana de Gerencia 22 (80): Pp. 639-56.

Molera, Lourdes, y Isabel P. Albaladejo. (2007), Profiling segments of tourists in rural areas of South-Eastern Spain. Tourism Management 28 (3): Pp. 757-67.

Mustelier, Lourdes, y Yureidys García. (2015), Contribución del turismo al desarrollo local en Cuba desde la perspectiva de la gestión del destino. Revista Científica ECOCIENCIA 2 (3): Pp. 1-21.

Narváez, Mercy, y Gladys Fernández. (2010), El turismo desde la perspectiva de la demanda. Lugar de estudio: península de Paraguaná - Venezuela. Divulgación Científica 13 (2): Pp. 175-83.

Narváez, Mercy, y Gladys Fernández. (2013), Redes de cooperación empresarial: relaciones e interacciones para promover desarrollo turístico local. Revista Venezolana de Gerencia 18 (61): Pp. 121-37.
Narváez, Mercy, Gladys Fernández, y Gutiérrez Carmen. (2018), Participación de las comunidades organizadas en el desarrollo turístico local (Paraguaná - Venezuela). Revista Venezolana de Gerencia 23 (81).

Oficina Nacional de Estadística Cuba. (2016), Anuario Estadístico Sancti Spíritus. Anuario Estadístico Sancti Spíritus. 2016. Recuperado en http:// www.one.cu/publicaciones/provincias_masinf/sancti\%20spiritus.htm Fecha de consulta: 13 de noviembre. 2017

Pardo, Campo, y Pedro Del Campo. (2007), Combinación de métodos factoriales y de análisis de conglomerados En R: El Paquete FactoClass. Revista Colombiana de Estadística 30 (2): Pp. 231-245.

Park, Duk Byeong, \& Yoo Shik Yoon. (2009), Segmentation by motivation in rural tourism: a Korean case study. Tourism Management 30 (1): Pp. 99-108.

Pérez, Edgardo, y Leonardo Medrano. (2010), Análisis factorial exploratorio: bases conceptuales y metodológicas. Revista Argentina de Ciencias del Comportamiento 2 (1): Pp. 58-66.

Pesonen, Juho Antti. (2014), Testing segment stability: insights from a rural tourism study. Journal of Travel and Tourism Marketing 31 (6): Pp. 697-711.

Prada-Trigo, José, Daniela Chillogallo, Abraham Córdova, y Leonardo León. (2018), El turista cultural: tipologías y análisis de las valoraciones del destino a partir del caso de estudio de Cuenca-Ecuador. Pasos. Revista de Turismo y Patrimonio Cultural 16 (1): Pp. 55-72.

Prado, Eduardo, y Jesús Mallou. (2000), Segmentando mercados con análisis conjunto. Una aplicación al sec- 
Segmentación y valoración del mercado turístico: Estudio del destino Patrimonio de la Humanidad Trinidad de Cuba

García Reinoso, Nelson

tor turístico. Psicothema 12 (2): Pp. 453-458.

Prebensen, Nina, Kåre Skallerud, \& Joseph S. Chen. (2010), Tourist motivation with sun and sand destinations: satisfaction and the wom-effect. Journal of Travel and Tourism Marketing 27 (8): Pp. 858-73.

Ramirez-Anormaliza, Richard, Fabricio Guevara-Viejo, Mayra Regnault, Rubi Pena-Holguin, Ruth Farias-Lema, Freddy Bravo-Duarte, José Díaz-Montenegro, et al. (2017), Análisis multivariante: teoría y práctica de las principales técnicas. Milagro, Ecuador: Holguín S.A-Universidad Estatal de Milagro - UNEMI.

Reinoso, Nelson. (2017), Modelo de gestión para fomentar el desarrollo turístico de las comunidades Manabitas, Ecuador. CULTUR - Revista de Cultura e Turismo 2: Pp. 81-108.

Reinoso, Nelson, y Neme Doumet. (2017), El producto turístico comunitario como estrategia para diversificar las economías locales del cantón Bolívar, provincia de Manabí, Ecuador. Revista Interamericana de Ambiente y Turismo 13 (1): Pp. 105-116.

Reinoso, Nelson, y Yomara Quintero. (2018), Producto de sol y playa para el desarrollo turístico del municipio Trinidad de Cuba. Revista Interamericana de Ambiente y Turismo (RIAT) 14 (1): Pp. 52-64.

Ricolfe, José S., Bernat Merino, Sara Marzo, María T. Ferrandis, y Cristina Rodríguez. (2008), Actitud hacia la gastronomía local de los turistas: dimensiones y segmentación de mercado. PASOS Revista de Turismo y Patrimonio Cultural 6 (2): Pp. 189-198.

Rid, Wolfgang, Ikechukwu O. Ezeuduji, \& Ulrike Pröbstl-Haider. (2014), Segmentation by motivation for rural tourism activities in the Gambia. Tourism Management 40 (2014): Pp. 102-116.

Rodríguez, J. Rosa, y Beatriz Abdul-Jalbar. (2012), Turismo cultural y planificación del viaje: un estudio de caso. Tourism \& Management Studies 8: Pp. 41-47.

Rodríguez, Orlando, y Teresa López. (2011), La Trinidad de Cuba: medio milenio de venturas y desventuras. Contribuciones a las Ciencias Sociales, 1-70.

Stylidis, Dimitrios, Jason Kokho Sit, \& Avital Biran. (2018), Residents' place image: a meaningful psychographic variable for tourism segmentation? Journal of Travel and Tourism Marketing 35 (6): Pp. 715-25.

Vaus, D. De. (2002), Analyzing social science data. London: Sage.

Vázquez de la Torre, María G., Luis Amador, y Juan M. Fuentes. (2016), La denominación de origen protegida 'Los Pedroches' como ruta gastronómica del jamón Ibérico: análisis del perfil del visitante y evolución futura. Cuadernos de Desarrollo Rural 13 (77): Pp. 63-91.

Vilà-Baños, Ruth, María J. Rubio-Hurtado, Vanesa Berlanga-Silvente, and Mercedes Torrado-Fonseca. (2014), Cómo aplicar un análisis jerárquico en SPSS. Revista d'Innovació i Recerca En Educació 7 (1): Pp. 113-27. 